\title{
Situación pesquera del centollón Paralomis granulosa, (Hombron \& Jacquinot, 1846) (Decapoda: Lithodidae) en Magallanes, Chile y consideraciones para mejorar el futuro manejo de la pesquería
}

Fisheries situation of the chilean snow crab Paralomis granulosa, (Hombron \& Jacquinot, 1846) (Decapoda: Lithodidae) in Magallanes, Chile and considerations to improve the future management of the fisherys

Eduardo Almonacid Rioseco ${ }^{1}$, Erik Daza Valdebenito ${ }^{1}$ \& Ruth Hernández Rodríguez ${ }^{1}$

En Chile, la pesquería de centollón (Paralomis granulosa, (Hombron \& Jacquinot 1846)) se inició en febrero de 1977 en la zona central del estrecho de Magallanes (Capodónico, 1977). Su distribución abarca desde Valdivia hasta el extremo sur de Sudamérica, incluyendo las islas Malvinas (Vinuesa et al. 1999). Su límite norte por el Atlántico, es la latitud $24^{\circ} 21^{\prime}$ S. Es una especie bentónica posible de encontrar entre los 10 y 150 $\mathrm{m}$ de profundad y generalmente se encuentra asociada a bosques de Macrocystis pyrifera (Castilla, 1985).

Esta actividad se desarrolla exclusivamente en Magallanes (zona austral de Chile) y Argentina, y encierra relativa importancia económica, si consideramos que es de carácter secundario con respecto a la pesquería de centolla (Lithodes santolla, Molina 1782). En algunos casos ha sido considerada una pesquería mixta debido a que ambas están estrechamente ligadas (Boschi et al. 1984; Boschi, 1997), además de ser considerada una actividad alternativa cuando $L$. santolla se encuentra en veda biológica (sensu Ley General de Pesca y Acuicultura $\mathrm{N}^{\circ}$ 21.033). No obstante, es especialmente importante en las inmediaciones de la isla Navarino (sur de Magallanes), principal sector de pesca de centollón del país (Fig. 1).

A más de cuarenta años de los primeros estudios acerca del centollón, la información referente al estado del recurso ha sido bastante exigua y con vacíos temporales, especialmente porque se ha otorgado más importancia a la centolla como recurso de mayor importancia económica y como principal objeto de estudio. En este contexto, la recopilación sistemática de información biológica pesquera del centollón se convierte en un pilar fundamental para su explotación y manejo.

En los últimos 28 años, su actividad muestra una reducción de los sectores de pesca en comparación con lo que ocurría en la década de los 80 s, donde se incluía al seno Otway $\left(53^{\circ} 00^{\prime}\right.$ $\left.\mathrm{S}-71^{\circ} 30^{\prime} \mathrm{O}\right)$, seno Keats (54 $19^{\prime} \mathrm{S}-70^{\circ} 41^{\prime} \mathrm{O}$ ),

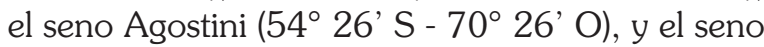
Martínez (54 $27^{\prime} \mathrm{S}$ - 70 40’ O) (Campodónico \& Hernández, 1981; Hernández, 1981, 1982), enfocándose actualmente en la Segunda Angostura del estrecho de Magallanes, principalmente en las inmediaciones de la isla Navarino como sectores de pesca habituales. Esto ha mantenido en operaciones a embarcaciones artesanales ligadas a los puertos de Punta Arenas, Porvenir y Puerto Williams, excluyendo a Puerto Natales donde se han registrado desembarques esporádicos (Daza et al. 2018). Esta pesquería ha sufrido fuertes fluctuaciones, principalmente condicionadas por la demanda de los mercados internacionales y por factores de tipo físico, lo que ha provocado intermitencias en el esfuerzo pesquero, cambios en el comportamiento del recurso y la condición de tamaño y abundancia.

La presente nota describe lo observado para un periodo de 11 años (2007-2017) a partir de información recabada por el Instituto de

Instituto de Fomento Pesquero.

Enrique Abello 0552, Punta Arenas, Chile.

$\measuredangle$ eduardo.almonacid@ifop.cl 

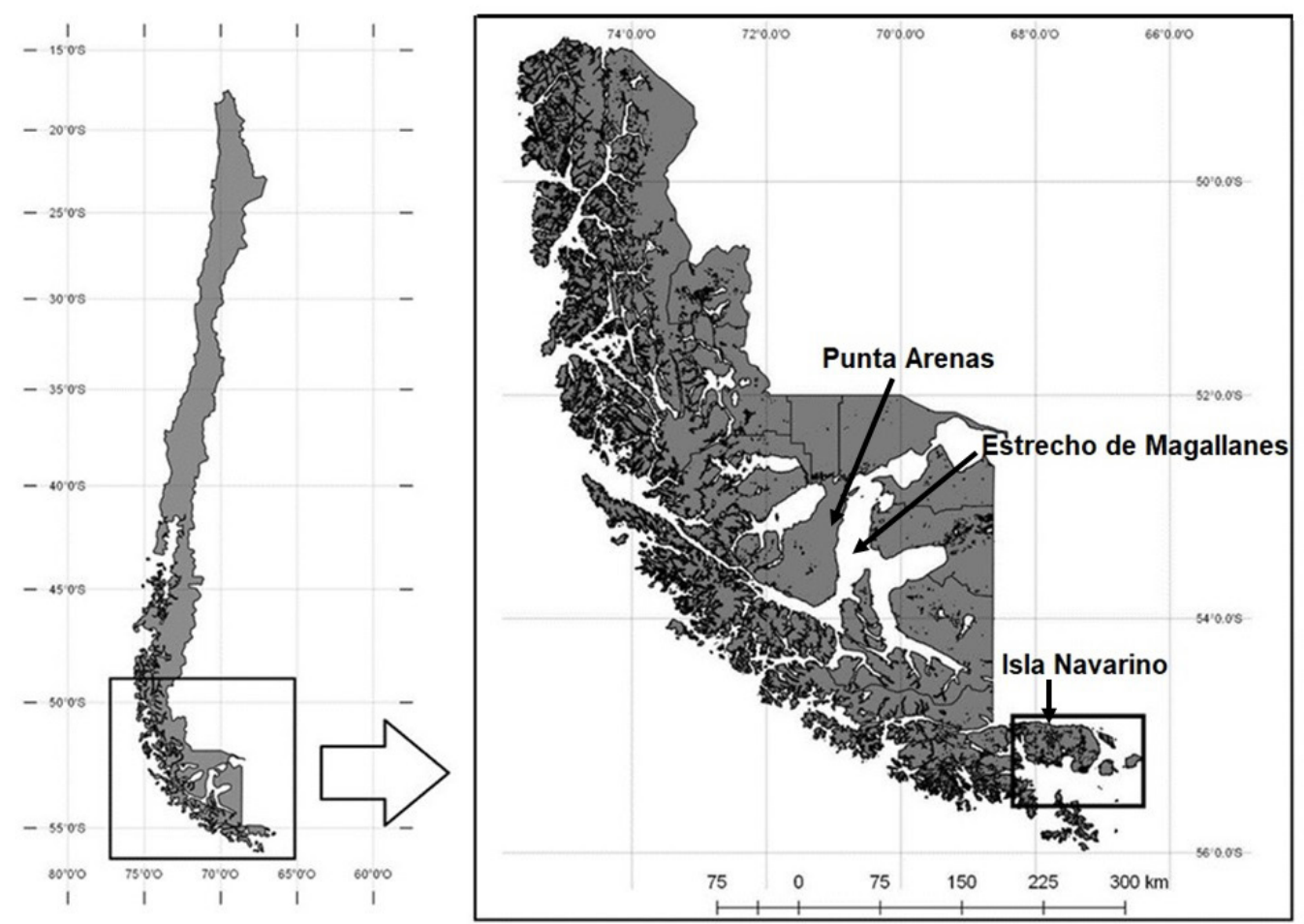

Fig. 1. Ubicación geográfica de la zona de Magallanes, isla Navarino y la zona de estudio (Daza et al. 2018).

Fomento Pesquero (IFOP) considerando datos de desembarque, talla mínima legal, fluctuaciones de los tamaños medios de machos y hembras en las capturas, proporciones de sexos y su relación con factores físicos. Lo anterior, teniendo en cuenta que existen otros factores no considerados y que bien podrían explicar por sí solas o ser complementarias tales fluctuaciones.

En Chile, la información bio-pesquera disponible se restringe a estudios realizados principalmente en la década de los 70s y 80s (Geagham, 1973; González \& Perugi, 1974; Sanhueza, 1976; Campodónico, 1980; Hernández, 1980, 1981, 1982; Campodónico \& Hernández, 1981; Inostroza et al. 1982; Hernández et al. 1984; Campodónico et al. 1983; Guzmán \& Ríos, 1985; Campodónico, 1986; Díaz, 1987; Campodónico et al. 1988), cuando presumiblemente el estado del recurso era más "saludable".

Salvo algunos estudios puntuales (Guzmán et al. 2004; Alarcón et al. 2007; Medina et al. 2017), en los siguientes 20 años se produjo un vacío de información que no permitió diagnosticar y contar con información para el manejo, control y desarrollo sostenible de esta pesquería.

Hasta 2017, se registró un total de 162 embarcaciones trabajando activamente en centolla y centollón, de las cuales un $25 \%$ se encontraban trabajando en ambos recursos (L. santolla y $P$. granulosa), un $54 \%$ se orientó a la centolla ( $L$. santolla) y un $21 \%$ al centollón ( $P$. granulosa), conformando un total aproximado de 120 pescadores artesanales dedicados exclusivamente a la actividad. Actualmente, aún se desconoce el número de pescadores informales que pudieran estar operando en ambas pesquerías (Daza et al. 2018).

$P$. granulosa cuenta con una regulación tipo SSS (sex, season, size; por sus siglas en inglés) que incluye utilización de trampas como arte de pesca, prohibición de desembarque y comercialización de hembras, talla mínima legal de captura de machos de $80 \mathrm{~mm}$ de longitud cefalotoráxica (LC), una temporada de pesca comprendida entre el 1 de febrero y el 30 de noviembre de cada año y sólo ingresar ejemplares machos vivos a las plantas de proceso. Además, se encuentra suspendida la inscripción de personas y embarcaciones en el 
Registro Pesquero Artesanal (RPA) del Servicio Nacional de Pesca (Sernapesca).

Según las estadísticas oficiales (Sernapesca, 2017), desde 1977 los desembarques de $P$. ganulosa fueron inferiores a los de L. santolla y hasta 1985 no superaban las $1.000 \mathrm{t}$, sin embargo, a partir de 1986 sufrieron un fuerte incremento, alcanzando en 1988 niveles por sobre las $2.000 \mathrm{t}$ y en 1991 sobrepasaron las 3.000 t. Entre 1992 y 1999 la captura de $P$. granulosa fue de alrededor de las $1.500 \mathrm{t}$ anuales, mientras que para los años 2000 y 2002 , los niveles de desembarque superaron cifras históricas sobrepasando las $6.500 \mathrm{t}$ (año 2001). Entre 2003 y 2004 se mantuvieron por el orden de las 3.234 t y en el año 2005 nuevamente hubo un repunte registrándose aproximadamente 6.000 t, no obstante, entre los años 2007 y 2016 se constató una drástica disminución del recurso alcanzando las 2.326 t en promedio. Esta cifra se vio aumentada notablemente para el año 2017 donde se alcanzaron las $5.936 \mathrm{t}$.

$\mathrm{Al}$ contrastar mensualmente las estadísticas oficiales de $P$. granulosa y $L$. santolla, se observó que a contar del año 2005 las mayores cifras correspondieron al primer semestre de cada año, especificamente entre febrero y junio. Posteriormente, durante el segundo semestre, el esfuerzo pesquero se orientó regularmente hacia el recurso alternativo (Fig. 2).

El análisis de los desembarques reflejó que los ejemplares bajo la talla mínima legal (BTML) mostraron valores relativamente altos, llegando el año 2011 a un $27 \%$, y aunque constituye una pesquería de menor importancia, según datos del Instituto de Fomento Pesquero (IFOP), a lo largo del tiempo estos valores han ido disminuyendo en forma progresiva, presumiblemente por un mayor énfasis en las medidas de control, fiscalización y auto regulación de la mayoría de los pescadores dedicados a la actividad (Fig. 3).

$\mathrm{Al}$ circunscribirnos en torno a las actividades pesqueras efectuadas en las inmediaciones de la isla Navarino como principal zona de pesca, en general se observó que las tallas medias de las capturas mostraron diferencias estacionales con una tendencia a capturar individuos de mayor talla en los periodos estivales. Esto reflejó lo descrito por Lovrich \& Vinuesa (1999), en el sentido que las poblaciones locales experimentan migraciones desde las zonas más profundas hacia zonas más someras con fines reproductivos en esa estación del año.

Así también, durante la temporada de pesca 2011 se observó que la talla media de los ejemplares machos fue inferior a las hembras durante la estación de invierno (Daza et al. 2018), situación catalogada como anormal, al ser comparada con el resto de las temporadas (Fig. 4).

Independientemente de la pesca, se deben tener presente que las perturbaciones provocadas

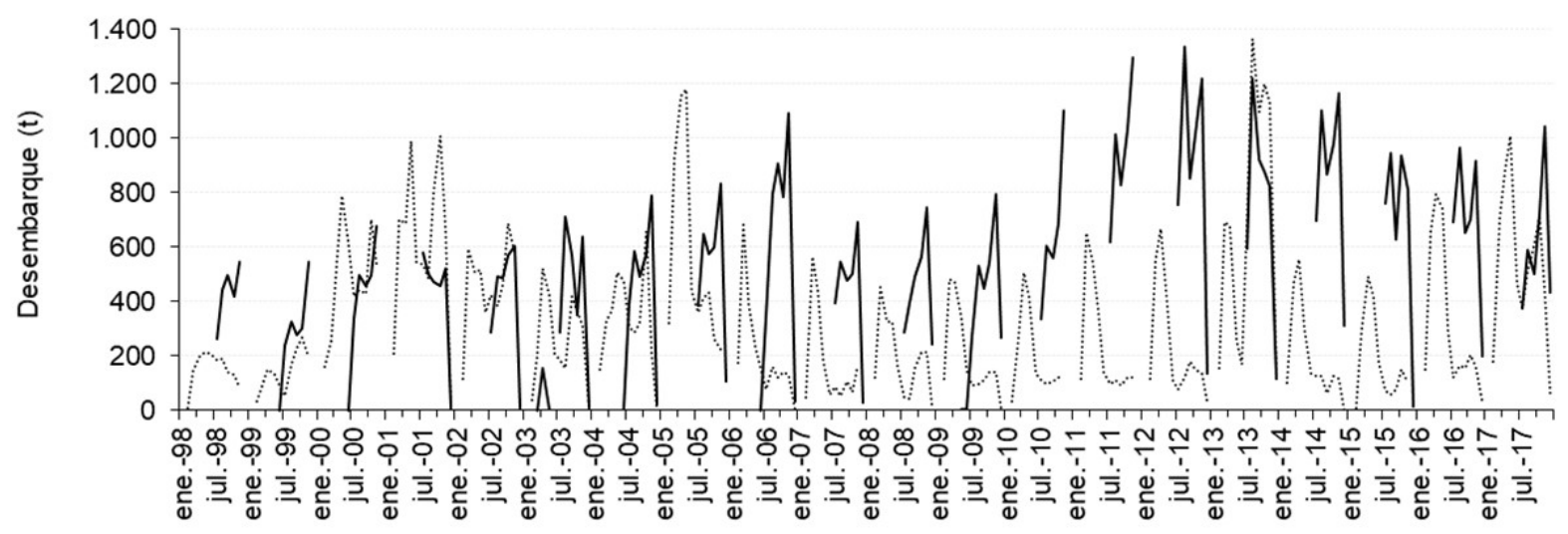

Periodo

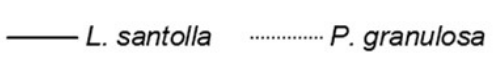

Fig. 2. Desembarques mensuales de los recursos Lithodes santolla y Paralomis granulosa en la región de Magallanes:

Periodo enero de 1998 a diciembre de 2017 (Sernapesca 2017). 


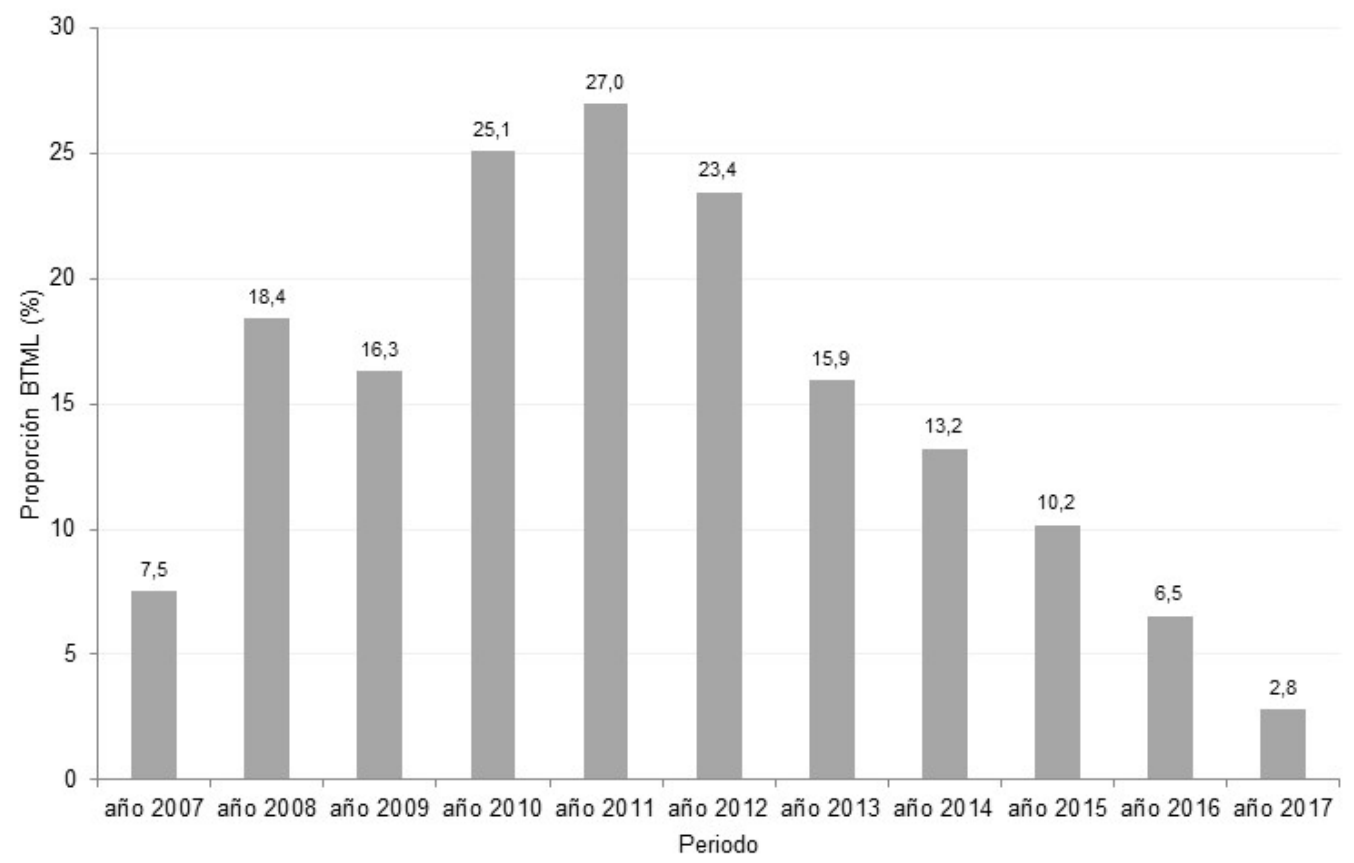

Fig. 3. Proporción de ejemplares de centollón bajo la talla mínima legal (BTML) entre el periodo 2007 y 2017 (Daza et al. 2018).

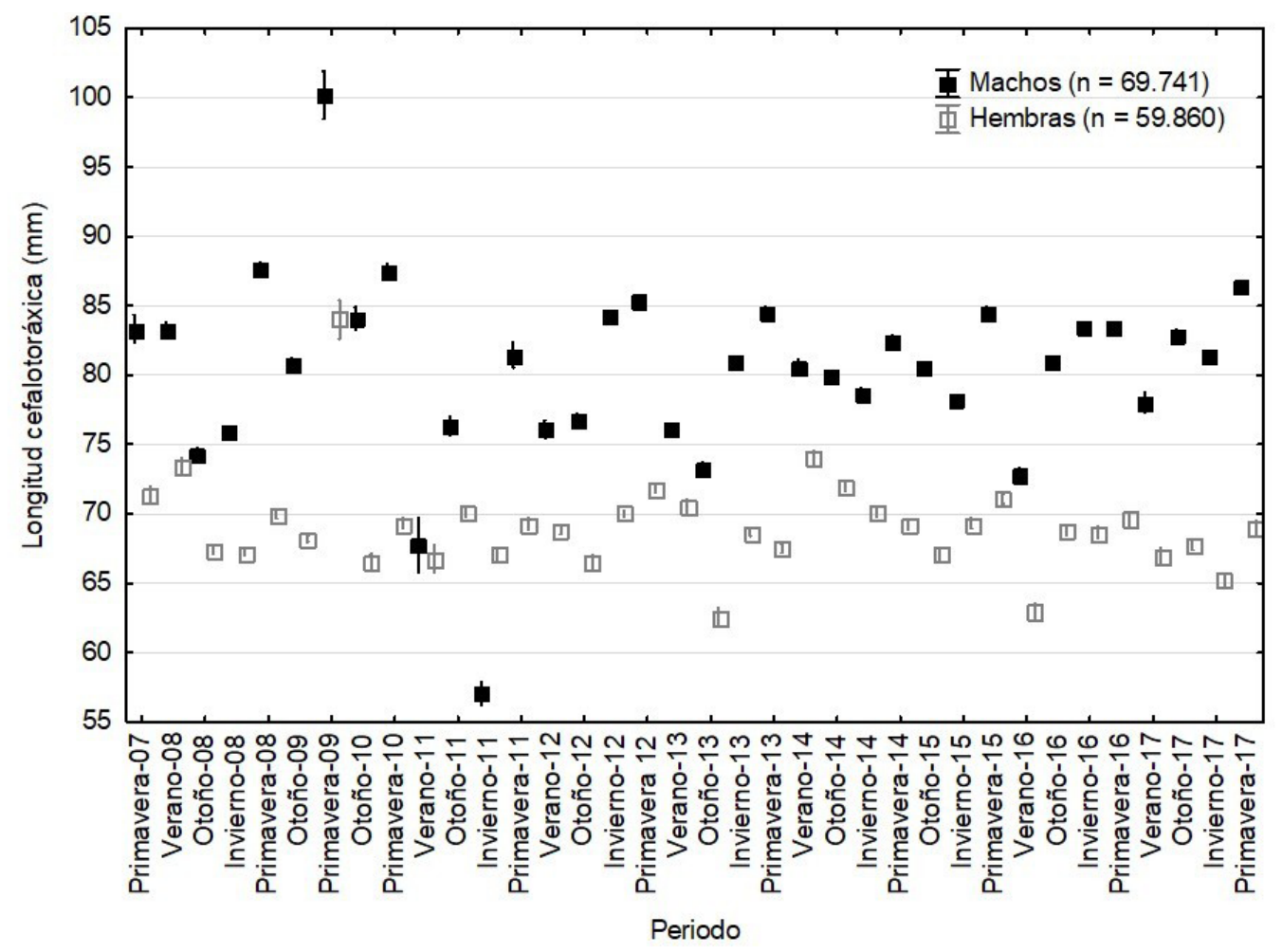

Fig. 4. Tallas medias ( \pm e.e.) estacionales de P. granulosa provenientes de sectores aledaños a la isla Navarino entre el periodo 2007 y 2017 (Daza et al. 2018). 
por alteraciones climáticas pueden provocar cambios en la abundancia de zooplancton y larvas de peces $u$ otros organismos que pueden constituir la dieta de estos crustáceos (Thatje et al. 2005). Es necesario integrar mediciones respecto a la temperatura superficial del agua de mar, porque es un factor preponderante y tiene una incidencia directa en los niveles de distribución y abundancia de los ejemplares comerciales (Almonacid \& Vargas, 2012). En el caso anterior, se estima que esto pudo ser el reflejo de una alteración en el reclutamiento, especialmente si consideramos que en la estación de invierno la temperatura superficial del mar se mantuvo constante alrededor de los $4,93^{\circ} \mathrm{C}$ en promedio (meses de julio y agosto), siendo el valor más bajo y extenso en el tiempo registrado entre 2007 y 2017 (https://giovanni.sci.gsfc.nasa.gov, 2018).

Los efectos que la disminución de la temperatura podría causar en estadios primarios, tienen relación con una tardía ovulación, desarrollo embriológico desacelerado y lento crecimiento larval (Petriella \& Boschi, 1997). En relación con los cambios en los periodos de florecimiento fitoplanctónico, estos factores podrían conducir a un desajuste entre la crianza de larvas y la presencia adecuada de presas, lo cual podría causar alteraciones en el reclutamiento y rango de distribución geográfica (Paschke et al. 2013).

A lo anterior, se suma que los niveles de abundancia relativa en términos de rendimientos nominales (CPUE) obtenidos durante 2011 se encontraban entre los más bajos del periodo 2007 a 2017 (Fig. 5). Posteriormente, se observó una progresiva tendencia hacia rendimientos mayores, lo que se explica porque la flota pesquera en esa zona ha ido operando a mayores profundidades, en áreas más expuestas al océano Pacífico, aumento en el poder de pesca, aumento en el esfuerzo pesquero $y$, por otra parte, por la experiencia y pericia de los patrones de pesca en las embarcaciones donde se registró la información, especialmente durante 2016 y 2017.

Durante el periodo analizado en este trabajo, la proporción de sexos no mostró un patrón claramente definido. En la mayoría de los casos, se observó una tendencia progresiva a capturar una menor proporción de machos y una mayor proporción de hembras (Alarcón et al. 2007). Así también, se observó una significativa correlación a lo largo de todas las temporadas de pesca respecto de la proporción de sexos en individuos maduros, a pesar que la relación no siempre fue de 1:1 ( $\mathrm{n}=79.523$; Coef. Corr. Spearman: 0,73; $p<0,05)$. Las más altas proporciones de machos $y$ hembras maduros fueron en los meses estivales (Daza et al. 2018).

En Magallanes se desconocen los sectores en donde ocurre la reproducción de centollón, aunque se estima que estos no se circunscriben a lugares específicos, sino que son de amplia distribución. Stevens (2014), plantea que pescar cerca de agregaciones de reproductores puede llegar a ser destructivo y contraproducente. Según este autor, muchas pesquerías efectúan sus actividades en áreas de apareamiento o desove bajo el supuesto que los machos se concentran ahí. En evidencia de esto, el cangrejo de Tanner (Chionoecetes bairdi) en el Pacífico norte refleja que las agregaciones de apareamiento pueden incluir a todas las hembras presentes en un radio de $50 \mathrm{~km}$ y su densidad no aumenta significativamente durante todo el periodo.

Stevens (2014), explica también que mientras mayor es la distancia del área de desove la presencia de los machos es menor. Esto sugiere que la actividad pesquera debiera ser prohibida dentro de las áreas de hembras desovantes y que las proporciones de sexos por si solas, no son información útil para el manejo, a menos que se conozca su posición geográfica específica. Así también, las hembras no pueden contribuir a futuras generaciones si ellas no están ubicadas en zonas apropiadas, de tal manera que en una pesquería como esta se puede obtener resultados indeseados.

Por su parte, Webb (2014) enumeró gran variedad de investigaciones sobre el género Lithodes, indicando que la remoción de cangrejos machos competitivamente dominantes puede perjudicar e impactar significativamente el éxito reproductivo, las tasas de fertilidad y el rendimiento reproductivo de las hembras. Por estas razones, además de incorporar la proporción de sexo/ tamaño también se necesita incluir la ubicación específica de estos ejemplares al momento de la explotación con el propósito de enfocarse en individuos no reproductivos y así reducir el 


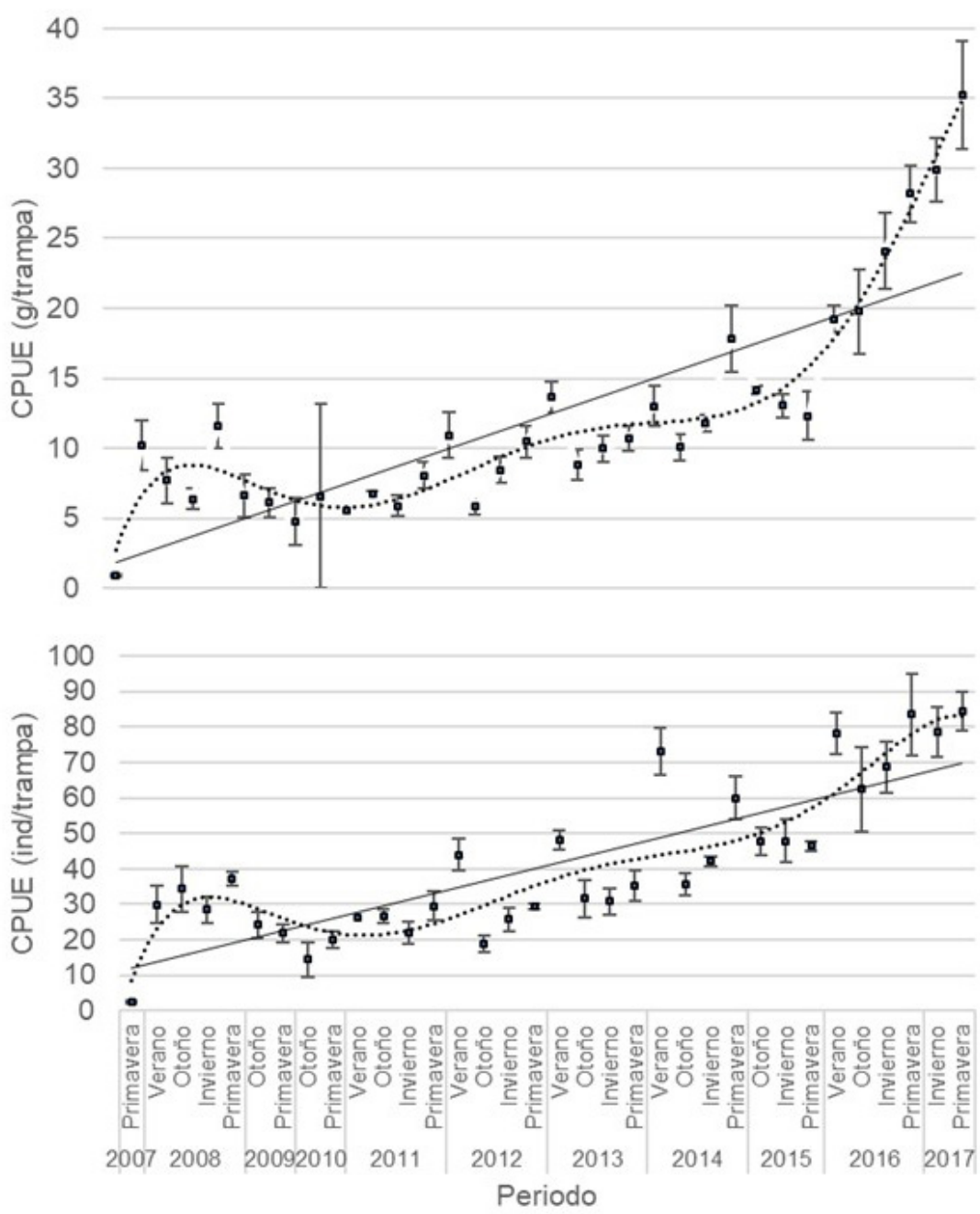

Fig. 5. Captura estacional por unidad de esfuerzo (CPUE) en términos de individuos/ trampa y kg/trampa de $P$. granulosa provenientes de sectores aledaños a la isla Navarino entre el periodo 2007 y 2017 (Fuente: Daza et al. 2018).

impacto en la reproducción. Esto en Magallanes aún se desconoce, aunque se sospecha que la o las poblaciones de centollón en el sistema de fiordos y canales son sustentadas a partir de las zonas oceánicas, principalmente.

Se ha observado una progresión en la estructura de tallas de machos en algunos sectores de los cuales se dispone de mayor información, aunque esta es incompleta dada la magnitud y extensión de los lugares donde habita. Como enfoque alternativo, la explotación balanceada de machos y hembras podría tener menos perjuicios sobre la diversidad marina si se conociera con precisión los periodos en los cuales se lleva a cabo la reproducción, que en Magallanes, debe responder a una gradiente latitudinal y longitudinal. Con este enfoque se busca nivelar la explotación basándose en remover igual proporción de machos y hembras.

Al remover solamente machos, disminuye la probabilidad de apareo durante el periodo de pesca, que por cierto se inicia aun cuando la pesquería se está ejecutando. En el corto plazo no es aplicable una explotación de este tipo, debido a que no se conocen los sectores en los cuales ocurre la reproducción. 
Es fundamental para la evaluación de este recurso conocer la variación latitudinal y longitudinal de la Talla de Madurez Sexual (TMS), actualizar los parámetros de crecimiento y realizar estudios poblacionales. Por otra parte, es necesario el seguimiento estratégico de los niveles de abundancia relativa (CPUE) e incorporar monitoreos de aspectos ambientales referidos a la ecología y comportamiento de la especie. Además, es importante un manejo integral de las pesquerías de litódidos en Magallanes, en donde la responsabilidad de los usuarios permita llevar a la práctica una explotación pesquera sustentable.

\section{AGRADECIMIENTOS}

Los autores desean agradecer al Instituto de Fomento Pesquero por el uso de la información perteneciente al "Programa de Seguimiento de las pesquerías de crustáceos bentónico. Centolla y centollón, Región de Magallanes y Antártica Chilena", así como también a la Subsecretaría de Pesca y Acuicultura (Gobierno de Chile) y el Ministerio de Economía, Fomento y Turismo. Se agradece además, el aporte de los revisores.

\section{LITERATURA CITADA}

Alarcón, R., Aedo, G., Galleguillos, R., Oyarzún, C., \& Veas, R. (2007). Investigación biológico pesquera recurso centolla y centollón, XII Región de Magallanes y Antártica Chilena. Informe Final. Ms.

Boschi, E. E., Bertuche, D. A., \& Wyngaard, J. G. (1984). Estudio biológico pesquero de la centolla (Lithodes antarcticus) del Canal Beagle, Tierra del Fuego. Mar del Plata: Contrib. INIDEP.

Boschi, E. (1997). Las pesquerías de crustáceos decápodos en el litoral de la república argentina. Investigaciones Mararinas, 25, 19-40.

Campodónico, I. (1980). Investigación, manejo y control de las pesquerías de centolla y centollón de la XII Región. Instituto de la Patagonia. Informe Final. Ms. Disponible en (http://www.bibliotecadigital.umag.cl)

Campodónico, I., \& Hernández, M. (1981). Investigación, manejo y control de las pesquerías de centolla y centollón de la XII Región (temporada 1980 - 81), Instituto de la Patagonia. Informe Final. Ms. Disponible en (http://www.bibliotecadigital.umag.cl)

Campodónico, I., Hernández, M. B., \& Riveros, E. (1983). Investigación, manejo y control de las pesquerías de centolla y centollón de la XII región. Informe consolidado: recurso centollón, Informe Instituto de la Patagonia. Ms. Disponible en (http://www. bibliotecadigital.umag.cl)_

Campodónico, I. (1986). Diagnóstico bio-pesquero de la centolla, XII región. Estudio en poblaciones de hembras, 1985. Informe Instituto de la Patagonia. Ms. Disponible en (http://www.bibliotecadigital.umag.cl)

Campodónico, I., \& J. López. (1988). Diagnóstico bio-pesquero de la centolla. XII región 1987. Estudio en poblaciones de hembras y crecimiento de juveniles en cautividad. Informe de proyecto. Instituto de Fomento Pesquero. Ms.

Díaz, P. (1987). Diagnóstico bio-pesquero del recurso centollón. IFOP- Chile. Ms.

Daza, E., Almonacid, E., \& Hernández, R. (2018). Programa de Seguimiento Pesquería Crustáceos Bentónicos en la Región de Magallanes. Informe Final. Subsecretaría de Economía. Ms.

Geagham, J. (1973). Resultados de las investigaciones sobre centolla, Lithodes antarctica (Jacquinot) realizadas por el Instituto de Fomento Pesquero en la provincia de Magallanes. Ms.

González, O., \& Perugi, M. (1974). Pesca exploratoria para detectar la presencia de centolla entre el estrecho de Magallanes y Puerto Natales. Instituto de Fomento Pesquero. Ms.

Guzmán, L., \& Ríos, C. (1985). Investigación, manejo y control de las pesquerías de centolla y centollón de la XII Región (1979-1983). Informe Consolidado: Recurso centolla (Lithodes antarcticus Jacquinot). Ms. Disponible en (http://www.bibliotecadigital. umag.cl)

Guzmán, L., Daza, E., Canales, C., Cornejo, S., Quiroz, J. C., \& González, M. (2004). Estudio biológico pesquero de centolla y 
centollón en la XII Región. Informe Final Instituto de Fomento Pesquero. Ms.

Hernández, M. B. (1981). Manejo de las pesquerías de centolla y centollón de la XII región. Segunda etapa 1980. Informe de proyecto. Instituto de Fomento Pesquero. Ms.

Hernández, M. B. (1982). Manejo de las pesquerías de centolla y centollón de la XII región. Primera etapa - 1979. Informe de proyecto. Instituto de Fomento Pesquero. Ms.

Hernández, M. B., Campodónico, I., \& Díaz, P. (1984). Investigaciones biológico-pesqueras del recurso centolla (Lithodes antarcticus) realizadas entre 1979 y 1984 en la XII región. Informe final, Instituto de Fomento Pesquero. Ms.

Inostroza, F., Gili, R., \& Salas, R. (1982). Programa de investigación de los recursos centolla, centollón y jaibas. Resultados del estudio. Instituto de Fomento Pesquero. Ms.

Lovrich, G. A., \& Vinuesa, J. H. (1999). Reproductive potential of the lithodids Lithodes santolla and Paralomis granulosa (Anomura, Decapoda) in the Beagle Chanel, Argentina. Scientia Marina, 63(supl. 1), 355-360.

Medina, A., Figueroa, T., \& Cañete, J. (2017). Caprella ungulina Mayer, 1903 (Amphipoda: Caprellidae): epizoo de Paralomis granulosa (Hombron \& Jacquinot, 1846) (Decapoda: Lithodidae) en aguas de Magallanes, Chile. Anales Instituto Patagonia, 45(1), 17-29

NASA. https://giovanni.sci.gsfc.nasa.gov. Última visita, septiembre 2018.

Paschke, K., Cumillaf, J., Chimal, M., Díaz, F.,
Gebauer, P., \& Rosas, C. (2013). Relationship between age and thermoregulatory behavior of Lithodes santolla (Molina, 1782) (Decapoda, Lithodidae) juveniles. Journal of Experimental Marine Biology and Ecology, 448, 141-145.

Petriella, A., \& Boschi, E. (1997). Crecimiento en crustáceos decápodos: resultados de investigaciones realizadas en Argentina. Investigaciones Marinas Valparaíso, 25, 135-157.

Sanhueza, A. (1976). Aspectos biológico-pesqueros del recurso centolla (Lithodes antarctica Jaquinot) de las áreas golfo Almirante Montt, seno Unión y canal Smyth. Serie Informes Pesqueros. Instituto de Fomento Pesquero. Ms.

Sernapesca. (2017). Anuario estadístico de pesca. www.sernapesca.cl. Última visita, octubre 2018.

Stevens, G. (2014). King crabs of the world: biology and fisheries management/ edited by Bradley G. Stevens.

Thatje, S., Anger, K., Calcagno, J., Lovrich, G., Pörtner, H., \& Arntz, E. (2005). Changelling de Cold: Crabs reconquer the Antarctic. Ecology, 86(3), 619-625.

Vinuesa, J. H., Lovrich, G. A. \& Tapella, F. (1999). New localities for Crustacea Decapoda in the Magellan region, southern South America. Scientia Marina, 63 (1), 321-323.

Webb. J. (2014). Reproductive Ecology of Commercially Important Lithdid Crabs. In Bradly G. Stevens (Ed.), King Crabs of the Word. Biology and Fisheries Management (pp. 285-314). 